\title{
Short term outcomes of preterm infants following antenatal corticosteroid treatment for childbearing women at $34(0 / 7)$ to $36(6 / 7)$ weeks: do the advantages outweigh the disadvantages?
}

\section{Zhimei Hu}

Second Affiliated Hospital of Shantou University Medical College

Xiaochun Deng

Second Affiliated Hospital of Shantou University Medical College

Honglin Liu

Second Affiliated Hospital of Shantou University Medical College

Lili Xie

Second Affiliated Hospital of Shantou University Medical College

Bingwei Liang

Second Affiliated Hospital of Shantou University Medical College

Jianhui Yang

Second Affiliated Hospital of Shantou University Medical College

Xuemei Lin

Second Affiliated Hospital of Shantou University Medical College

\section{Peishan Chen}

Second Affiliated Hospital of Shantou University Medical College

\section{Yuejun Huang ( $\nabla$ moon_hyj@qq.com )}

Second Affiliated Hospital of Shantou University Medical College https://orcid.org/0000-0001-82405738

\section{Research article}

Keywords: antenatal corticosteroids; late preterm birth; short-term outcomes; preterm infants

Posted Date: August 10th, 2020

DOI: https://doi.org/10.21203/rs.3.rs-27922/v2

License: (9) (1) This work is licensed under a Creative Commons Attribution 4.0 International License. Read Full License 


\section{Abstract}

\section{Background}

The effects of maternal antenatal corticosteroid (ACS) treatment, for fetal maturation, on the short-term outcome of late preterm infants are unclear.

\section{Methods}

This is a retrospective cohort study conducted in the Second Affiliated Hospital of Shantou University Medical College. Data of pregnant women who gave birth between $34(0 / 7)$ to $36(6 / 7)$ weeks gestation from January 2014 to June 2019 were collected. Nine short-term outcomes of preterm infants from mothers who received ACS treatment were compared to preterm infants from mothers who did not receive ACS treatment.

\section{Results}

In total, 1393 pregnant women (of whom 757 accepted ACS treatment before delivery) and 1472 preterm infants were eligible for analysis. The results were as follows: (1) ACS administration to pregnant women at high risk for giving birth between $34(0 / 7)$ to $36(6 / 7)$ weeks pregnancy, was related to decreasing cost and infant inpatient time (slope was $-0.784, P=0.026$ and slope was $-933.173, P=0.001$, respectively); ( 2 ) lack of maternal ACS treatment was an independent risk factor for neonatal respiratory distress syndrome (RR=0.548, $95 \% \mathrm{Cl}=0.332 \varangle 0.906)$; (3) use of maternal ACS did not increase risk of neonatal pneumonia, neonatal hypoglycemia, neonatal sepsis, necrotizing enterocolitis of newborns, neonatal intracranial hemorrhage, and hypoxic-ischemic encephalopathy in preterm infants.

\section{Conclusions}

Use of ACS for pregnant women at risk for giving birth between $34(0 / 7)$ to $36(6 / 7)$ weeks pregnancy may have more advantages than disadvantages. Our study provides evidence-based medicine for clinicians to make ACS treatment choices for pregnant women with risk of giving birth between $34(0 / 7)$ to $36(6 / 7)$ weeks gestation.

\section{Background}

Preterm birth is the second highest cause of child death in children younger than 5 years [1]. In 2010, an estimated 14.9 million babies (uncertainty range 12.3-18.1 million) were born preterm, comprising $11.1 \%$ of all livebirths worldwide [2]. Late preterm birth is defined as infants born between 34囚0/7区to $36(6 / 7)$ weeks gestation and accounts for $70 \%$ of all preterm births [3]. Recent studies show that even babies born at late preterm have an increased risk of immediate complications [4], neonatal and infant death, cerebral palsy, and worse neurodevelopmental and school performance outcomes when compared with those born at term [5]. 
In the last few decades, antenatal corticosteroid (ACS) treatment has been administered to childbearing women who are at risk for giving birth before 34 weeks, and has achieved tremendous success in reducing adverse neonatal outcomes, especially for preventing respiratory morbidity in preterm neonates [6]. However, such treatment has not been extended, to women during late preterm, because of a lack of consensus concerning ACS treatment for women who are at risk for giving birth between $34(0 / 7)$ to 36 (6/7) weeks gestation [7]. There are positive and negative views on ACS treatment for women at high risk of giving birth at $34(0 / 7)$ to $36(6 / 7)$ weeks. The positive view indicates that ACS treatment can decrease incidence of neonatal respiratory distress syndrome (NRDS) and neonatal hospitalization expenses in preterm infants between $34(0 / 7)$ to $36(6 / 7)$ weeks gestation [8-10]. The negative view indicates that this treatment does not reduce respiratory disease and wet lung in late preterm infants, but increases incidence of neonatal hypoglycemia and neonatal sepsis [11, 12]. The purpose of this study is to compare the advantages and disadvantages of preterm infant short-term outcomes of ACS treatment for pregnant women of $34(0 / 7)$ to $36(6 / 7)$ weeks.

\section{Methods}

\section{Setting and participants}

This is a retrospective cohort study. The study protocol was approved by the research institute's committee of human research in the Second Affiliated Hospital of Shantou University Medical College (N0.2018-23) and abided by the standards of the Declaration of Helsinki. The data were anonymized in this study, so we did not use the consent to participate.

We collected data of pregnant women who gave birth at $34(0 / 7)$ to $36(6 / 7)$ weeks gestation from January 2014 to June 2019. Data were excluded from this study if the pregnant women or preterm infants met the following criteria: (1) pregnant women had serious liver, kidney, lung or heart disease before or during pregnancy, (2) pregnant women received ACS treatment before 34 gestational weeks, (3) preterm infants had a congenital malformation or needed surgery.

\section{Data collection}

Variables included age of mother, gestational diabetes mellitus, pregnancy hypertension, method of delivery, premature rupture of membranes (PROM), condition of the placenta, meconium-stained amniotic fluid, multiple gestation, gestational age, birth weight of preterm infants, asphyxia, pulmonary surfactant treatment for preterm infants, and mechanical ventilation for preterm infants. The short-term outcomes of preterm infants in this study included the length of neonatal hospital stay, hospitalization expenses for preterm infants, NRDS, neonatal pneumonia, neonatal hypoglycemia, neonatal sepsis, necrotizing enterocolitis of newborn, neonatal intracranial hemorrhage, and hypoxic-ischemic encephalopathy.

\section{Exposure factor in this cohort is ACS treatment}


The patient cohort was divided into two groups: the ACS group and the without-ACS group. Antenatal corticosteroid treatment consisted of four doses of dexamethasone (6 mg intramuscular) 12 hours apart [13]. Whether to use antenatal corticosteroid after 34 weeks of preterm delivery or not was based on the choice of the pregnant women.

\section{Assessment short-term outcomes of preterm infants}

We compared nine short-term outcomes of preterm infants between the ACS group and without-ACS group. Complications of premature infants were diagnosed by doctors in the Neonatal Department, who did not know we were going to conduct this retrospective study when they made diagnosis. All doctors in the Neonatal Department made the diagnosis of complications according to standard diagnostic criteria. Assessment methods of these outcomes is described below. Neonatal hypoglycemia was defined as a glucose level less than $2.2 \mathrm{mmol}$ per liter [14].

A diagnosis of NRDS was based upon the findings of respiratory difficulty (cyanosis, grunting, nasal flaring, or tachypnea) that necessitated mechanical ventilation support, and was furthermore consistent with typical radiological findings of the lung (such as frosted glass-like changes, air bronchogram, and white lung). Laboratory findings were characterized initially by hypoxemia and later by progressive hypoxemia, hypercapnia, and variable metabolic acidosis. The clinical course, chest $x$-ray findings, and blood gas and acid-base values helped to establish the clinical diagnosis of NRDS [15].

Diagnosis of neonatal sepsis was based on symptoms and laboratory evidence. Initial symptoms might be few, and included apnea, tachypnoea, or tachycardia. Late complications of neonatal sepsis might include respiratory failure, pulmonary hypertension, cardiac failure, shock, renal failure, liver dysfunction, cerebral edema or thrombosis, adrenal hemorrhage or insufficiency, bone marrow dysfunction, and disseminated intravascular coagulation. Neonatal sepsis can be diagnosed when blood or other sterile body site culture produces positive pathogenic bacteria or opportunistic pathogens. The inflammatory response was measured by blood count, C-reactive protein, procalcitonin, interleukin 6 , interleukin 8 , and tumor necrosis factor. [16].

Gastrointestinal signs of necrotizing enterocolitis included feeding difficulty, gastric retention, abdominal distension, bilious vomiting, and stool with blood [17]. Necrotizing enterocolitis of newborn was defined according to Bell's criteria $\geq$ stage $2 \mathrm{~A}$. Diagnosis was made based on plain abdominal radiographs. A finding of pneumatosis in the intestinal wall confirmed the clinical suspicion and diagnosis of necrotizing enterocolitis of newborn.

Neonatal pneumonia was inflammation of the lung caused by infection. Diagnosis of neonatal pneumonia was made according to the risk factors, such as PROM, chorioamnionitis in the mother, and low birthweight, which predispose to pneumonia. Infants with respiratory distress usually required investigation to identify infection. A chest X-ray, showing increased bronchovascular shadows with small patchy and macular shadows helped to diagnose neonatal pneumonia [18]. 
Intracranial hemorrhage was suspected on the basis of the history, clinical manifestations, and knowledge of the birthweight-specific risks for intravascular hemolysis. Ultrasonography was the preferred imaging technique for screening. All at-risk infants underwent cranial ultrasonography within the first 3-7 days of age [19].

A diagnosis of hypoxic-ischemic encephalopathy was made according to the $\mathrm{pH}$ value of the fetal umbilical artery, the Apgar score at 5 and 10 minutes, and multi-system organ failure, including combined kidney damage, liver damage, blood abnormalities, heart dysfunction, metabolic disorders, and gastrointestinal tract injury [20]. Magnetic resonance imaging was used to evaluate extensive periventricular injury. All at-risk preterm infants underwent an MRI within the first week of age.

Because this is a single center study, we compared the neonatal hospitalization expenses directly. Hospitalization expenses included the examination, drug, and nursing care costs. Length of hospital stay was counted from the day of admission to the day of discharge.

\section{Statistical analysis}

We used the Shapiro-Wilk test to determine whether continuous variables were normally distributed, and the Wilcoxon-Mann-Whitney U-test was conducted for skewed distributions (presented as the median and the min-max range). Descriptive statistics for categorical variables are shown as frequency (percentage). The Pearson chi-square test or Fisher's exact test were used to compare categorical variables, as appropriate. Collinearity among all covariates was assessed using the Spearman correlation test [21].

For binary variables, logistic regression was used to analyze the risk factors of neonatal short-term outcomes, and independent variables were chosen based on clinical knowledge. For continuous dependent variables, linear regression was used to analyze the relationship between independent variables. Regression analysis was performed by a forward stepwise method to identify the risk factors. Estimated slope and $95 \%$ confidence intervals $(\mathrm{Cl})$ were obtained. Statistical analyses were performed using SPSS 24.0 (SPSS, Chicago, IL). P-values of less than 0.05 were considered to be statistically significant.

\section{Results}

\section{Population characteristics}

In total, 1393 pregnant women and 1472 preterm infants were eligible for analysis. Two preterm triplets and 73 preterm twins were included in this study. Seven hundred fifty-seven out of the 1393 pregnant women (54.3\%) accepted ACS treatment before giving birth. We analyzed the patient characteristics and clinical variables with and without maternal ACS treatment in this cohort (see Table 1). Women who were at higher maternal age, at low gestational age, developed PROM before delivery, or had parturition by cesarean section were more likely to receive ACS treatment. 
In order to compare the advantages and disadvantages of preterm infant short-term outcomes of ACS treatment in pregnant women at $34(0 / 7)$ to $36(6 / 7)$ weeks, we analyzed the risk factors for neonatal hypoglycemia, NRDS, neonatal sepsis, necrotizing enterocolitis of newborn, neonatal pneumonia, neonatal intracranial hemorrhage, hypoxic-ischemic encephalopathy, length of neonatal hospital stay, and hospitalization expenses of preterm infants (see Table 2).

The risk factors for neonatal hypoglycemia in this cohort were higher maternal age ( $R R=1.027$, $95 \% \mathrm{Cl}=1.002 \otimes 1.054)$ and low gestational age $(\mathrm{RR}=0.686,95 \% \mathrm{Cl}=0.580 \otimes 0.812)$. The risk factors for neonatal sepsis in preterm infants were vaginal delivery $(\mathrm{RR}=0.709,95 \% \mathrm{Cl}=0.848 \otimes 1.233)$, low birth weight $(\mathrm{RR}=0.541,95 \% \mathrm{Cl}=0.380 \rrbracket 0.772)$, NRDS ( $\mathrm{RR}=3.203,95 \% \mathrm{Cl}=1.780 \otimes 5.763)$, neonatal pneumonia $(\mathrm{RR}=1.788$, $95 \% \mathrm{Cl}=1.124 \rrbracket 2.846)$, necrotizing enterocolitis of newborn ( $\mathrm{RR}=3.009,95 \% \mathrm{Cl}=1.177 \rrbracket 7.692)$, and the mother with PROM (RR=2.824, 95\% Cl=2.090冈3.816). The risk factors for necrotizing enterocolitis of newborn in preterm infants were low gestational age $(\mathrm{RR}=0.381,95 \% \mathrm{Cl}=0.211 \times 0.688)$ and neonatal sepsis $(\mathrm{RR}=2.562,95 \% \mathrm{Cl}=1.023 \otimes 6.420)$. The risk factors for neonatal pneumonia in preterm infants were higher maternal age $(R R=1.049,95 \% \mathrm{Cl}=1.009 \otimes 1.091)$, lower gestational age $(R R=0.381,95 \% \mathrm{Cl}=0.211 \rrbracket$ $0.688)$, NRDS ( $R R=2.140,95 \% \mathrm{Cl}=1.005 \rrbracket 4.558)$, and asphyxia $(\mathrm{RR}=2.157,95 \% \mathrm{Cl}=1.164 \rrbracket 3.996)$. The risk factors for neonatal intracranial hemorrhage in preterm infants were vaginal delivery $(R R=0.320$, $95 \% \mathrm{Cl}=0.151 \varangle 0.679)$, low birth weight $(\mathrm{RR}=2.493,95 \% \mathrm{Cl}=1.155 \rrbracket 5.379)$, NRDS $(\mathrm{RR}=3.183,95 \% \mathrm{Cl}=1.441 \rrbracket$ 7.029), and asphyxia ( $\mathrm{RR}=2.976,95 \% \mathrm{Cl}=1.162 \bigotimes 7.624)$, and the risk factors for hypoxic-ischemic encephalopathy in preterm infants were NRDS $(R R=2.628,95 \% \mathrm{Cl}=1.749 \llbracket 3.948)$ and asphyxia $(\mathrm{RR}=9.714$, $95 \% \mathrm{Cl}=6.259815 .076)$. The above results show use of ACS treatment was not a risk factor of neonatal hypoglycemia, necrotizing enterocolitis of newborn, neonatal sepsis, neonatal pneumonia, neonatal intracranial hemorrhage, and hypoxic-ischemic encephalopathy.

The risk factors for NRDS in preterm infants were low gestational age ( $R R=0.529,95 \% \mathrm{Cl}=0.387 \otimes 0.724)$ and lack of ACS treatment ( $R R=0.548,95 \% \mathrm{Cl}=0.332 \varangle 0.906)$, which indicates that the incidence of NRDS in preterm infants whose mother received ACS treatment before delivery was about $50 \%$ that of preterm infants whose mothers did not use ACS (see Table 2).

Linear regression was used to analyze the length of hospital stay and the hospitalization expenses for preterm infants. According to linear regression analysis, a longer hospital stay of preterm infants was related to a lower gestational age, lack of maternal ACS treatment, mechanical ventilation for preterm infants, NRDS, neonatal hypoglycemia, neonatal pneumonia, neonatal sepsis, and hypoxic-ischemic encephalopathy (see Table 3). Longer hospital stay, use of pneumonia surfactant, mechanical ventilation for preterm infants, lower birth weight, lower gestational age, NRDS, neonatal pneumonia, neonatal hypoxic-ischemic encephalopathy, and lack of maternal ACS treatment were correlated with more neonatal hospital expenses (see Table 4). Maternal ACS treatment was negatively correlated with the length of hospital stay and the hospitalization expenses of preterm infants (slope was $-0.784, P=0.026$ and $-933.173, P=0.001$, respectively). The above results suggest that ACS administration to pregnant women who had a high risk for giving birth between $34(0 / 7)$ to $36(6 / 7)$ weeks gestation is associated with decreasing cost and infant inpatient time. 


\section{Discussion}

We find that ACS treatment is related to decreasing the incidence of NRDS, length of neonatal hospital stay, and neonatal hospital expenses, which infer that later preterm infants whose mothers received ACS recover better or faster from disorders Although some studies report that administration of ACS in the late preterm period could decrease the incidence of NRDS or respiratory disease $[8,9]$, the sample sizes in those studies were smaller, some of these studies only used single factor analysis, and some did not consider the influence of asphyxia and neonatal hypoglycemia on the incidence of NRDS [9]. There is a prospective study concerning ACS treatment during the late preterm period that compares neonatal primary outcomes with and without ACS treatment, and that included NRDS, neonatal sepsis, respiratory morbidity, neonatal death, and neonatal hypoglycemia. It was found that ACS treatment could increase the incidence of neonatal hypoglycemia and neonatal sepsis [11]. Another RCT study found that use of ACS for pregnant women who give birth at $34(0 / 7)$ to $36(6 / 7)$ weeks gestation could increase the incidence of neonatal hypoglycemia [8]. However, there were only 74 pregnant women in the ACS group in the first study, and moreover, they did not control for the influence of preterm infant birth weight on neonatal hypoglycemia and asphyxia in the study. In our study, ACS treatment for pregnant women at high risk of giving birth between $34(0 / 7)$ to $36(6 / 7)$ weeks gestation did not increase the risk of neonatal hypoglycemia and neonatal sepsis after controlling for other confounders and interactions between diseases. The above findings are consistent with prior research as follows. A large randomized trial study concerning ACS treatment, involving 1427 ACS-treated and 1400 placebo-treated pregnant women who gave birth at $34(0 / 7)$ to $36(6 / 7)$ weeks gestation, found ACS use could decrease the incidence of severe respiratory complications of preterm infants, but had no influence on the use of mechanical ventilation for preterm infants and the incidence of neonatal sepsis [8].

Maternal ACS treatment for pregnant women who give birth at $34(0 / 7)$ to $36(6 / 7)$ weeks gestation is still controversial. The Society for Maternal-Fetal Medicine recommends ACS treatment for women with a singleton pregnancy between $34(0 / 7)$ to $36(6 / 7)$ weeks gestation, who are at high risk for giving birth, to decrease incidence of NRDS and use of mechanical ventilation for preterm infants [22]. The American College of Obstetricians and Gynecologists recommends a single course of betamethasone for pregnant women between $34(0 / 7)$ to $36(6 / 7)$ weeks of gestation, and at risk of giving birth within 7 days, who had not received a prior course of ACS treatment [23]. The Society of Obstetricians and Gynecologists of Canada suggests that possible neonatal benefit should be weighed against possible long-term harm when considering ACS at 35 to 36 weeks gestation [24]. In this cohort study, we find maternal ACS is related to decreasing the incidence of NRDS, length of neonatal hospital stay, and neonatal hospital expenses. Although neonatal hospital stay and neonatal hospital expenses in the ACS group were higher than the without-ACS group, preterm infants in the ACS group had a higher rate of cesarean section, higher incidence of PROM, lower preterm gestational age, and lower body weight, which may be the reason for the increased hospitalization time and hospitalization cost for the preterm infants. Therefore, we used linear regression to correct the effect of the above patient characteristics on the hospitalization time and hospitalization cost in the ACS group vs. without ACS group. The results showed that neonatal hospital stay and neonatal hospital expenses for the ACS group are lower than for the without-ACS group 
after controlling for the confounding factors. Moreover, we found use of ACS treatment for pregnant women who gave birth between $34(0 / 7)$ and 36(6/7) weeks gestation does not increase the risk of neonatal pneumonia, neonatal hypoglycemia, neonatal sepsis, necrotizing enterocolitis of newborn, neonatal intracranial hemorrhage, and hypoxic-ischemic encephalopathy in preterm infants. Therefore, we believe that use of ACS for pregnant women giving birth at 34 to $36(6 / 7)$ gestation has more advantages than disadvantages. However, this is a cohort study, which may be inherently biased within group allocation.

\section{Conclusion}

In this study, we found use of ACS, for pregnant women who gave birth between $34(0 / 7)$ to $36(6 / 7)$ gestation, is associated with decreasing the incidence of NRDS, length of neonatal hospital stay, and neonatal hospital expenses, but has no adverse effect on the incidence of neonatal complications, which are the major risk factors for the long-term outcomes of preterm infants. Because the possible neonatal benefit of maternal ACS treatment could weigh against the possible long-term harm for preterm infants, we suggest that pregnant women with late preterm labor should undergo ACS treatment. Our study provides evidence-based medicine for clinicians to make ACS choices for pregnant women with high risk of giving birth between $34(0 / 7)$ to $36(6 / 7)$ gestation.

\section{Abbreviations}

ACS: antenatal corticosteroids; GDM: gestational diabetes mellitus; NRDS: neonatal respiratory distress; PROM: premature rupture of membranes.

\section{Declarations}

\section{Ethics approval and consent of participate}

The study protocol was approved by the research institute's committee of human research in the Second Affiliated Hospital of Shantou University Medical College (NO.2018-23) and abided by the standards of the Declaration of Helsinki. This is a retrospective cohort study. The data were anonymized in this study, so we did not use the consent to participate.

\section{Consent for publication}

Not applicable.

\section{Availability of data and material}

The data in this study are available from the corresponding author on reasonable request.

\section{Competing interests}


The authors declare that they have no competing interests in our study.

\section{Funding}

This research was supported by Science and Technology Planning Project of Guangdong Province (Grant Number: Shantou city government science and technology [2019]113-53 and [2020]113-118), and Li Ka Shing Foundation Cross-Disciplinary Research Grant (Project Number: 2020LKSFG03B).

The above mention funders had no role in the study design, data collection and analysis, decision to publish, or preparation of the manuscripts.

\section{Author contributions}

ZMH performed statistical analysis and wrote the manuscripts. XCD, HLL, LLX, and BWL collected the data of mother. JHY and XML collected the data of preterm infants. PSC and YJH contributed in experiment design and reviewing the final manuscripts. The author read and approved the final manuscripts.

\section{Acknowledgments}

We gratefully recognize Prof. Stanley Lin in Shantou University Medical College for language help.

\section{References}

1. Liu L, Johnson HL, Cousens S, Perin J, Scott S, Lawn JE, Rudan I, Campbell H, Cibulskis R, Li M et al: Global, regional, and national causes of child mortality: an updated systematic analysis for $\mathbf{2 0 1 0}$ with time trends since 2000. The Lancet 2012; 379(9832):2151-2161.

2. Blencowe H, Cousens S, Oestergaard MZ, Chou D, Moller A, Narwal R, Adler A, Vera Garcia C, Rohde S, Say $L$ et al: National, regional, and worldwide estimates of preterm birth rates in the year $\mathbf{2 0 1 0}$ with time trends since 1990 for selected countries: a systematic analysis and implications. The Lancet 2012; 379(9832):2162-2172.

3. Russell Rebecca B,Green Nancy S,Steiner Claudia A et al: Cost of hospitalization for preterm and low birth weight infants in the United States. Pediatrics 2007; 120: e1-9.

4. Consortium on Safe Labor,Hibbard Judith U,Wilkins Isabelle et al: Respiratory morbidity in late preterm births. JAMA 2010; 304: 419-425.

5. Petrini JR, Dias T, McCormick MC, Massolo ML, Green NS, Escobar GJ: Increased Risk of Adverse Neurological Development for Late Preterm Infants. The Journal of Pediatrics 2009; 154(2):169-176.

6. NIH Consensus Development Panel on the Effect of Corticosteroids for Fetal Maturation on Perinatal Outcomes: Effect of corticosteroids for fetal maturation on perinatal outcomes. JAMA 1995; 273(5): 413-418. 
7. Shanks AL, Grasch JL, Quinney SK, Haas DM: Controversies in antenatal corticosteroids. Seminars in Fetal and Neonatal Medicine 2019; 24(3):182-188.

8. Gyamfi-Bannerman C, Thom EA, Blackwell SC, Tita AT, Reddy UM, Saade GR, Rouse DJ, McKenna DS, Clark EA, Thorp JJ et al: Antenatal Betamethasone for Women at Risk for Late Preterm Delivery. N Engl J Med 2016; 374(14):1311-1320.

9. Balci O, Ozdemir S, Mahmoud AS, Acar A, Colakoglu MC: The Effect of Antenatal Steroids on Fetal Lung Maturation between the 34th and 36th Week of Pregnancy. GYNECOL OBSTET INVES 2010; 70(2):95-99.

10. Gyamfi-Bannerman C, Zupancic J, Sandoval G, Grobman WA, Blackwell SC, Tita A, Reddy UM, Jain L, Saade GR, Rouse DJ et al: Costeffectiveness of Antenatal Corticosteroid Therapy vs No Therapy in Women at Risk of Late Preterm Delivery: A Secondary Analysis of a Randomized Clinical Trial. JAMA PEDIATR 2019; 173(5):462-468.

11. Ramadan MK, Hussein G, Saheb W, Rajab M, Mirza FG: Antenatal corticosteroids in the late preterm period: A prospective cohort study. J Neonatal Perinatal Med 2016; 9(1):15-22.

12. Porto AM, Coutinho IC, Correia JB, Amorim MM: Effectiveness of antenatal corticosteroids in reducing respiratory disorders in late preterm infants: randomised clinical trial. BMJ 2011; 342: d1696.

13. Huddleston JF, Sanchez-Ramos L, Huddleston KW: Acute management of preterm labor. CLIN PERINATOL 2003; 30(4):803-824.

14. Arya Ved Bhushan,Senniappan Senthil,Guemes Maria et al: Neonatal Hypoglycemia. Indian Journal of Pediatrics 2014; 81(1):58-65.

15. Sweet DG, Carnielli V, Greisen G, Hallman M, Ozek E, Plavka R, Saugstad OD, Simeoni U, Speer CP, Vento $\mathrm{M}$ et al: European Consensus Guidelines on the Management of Neonatal Respiratory Distress Syndrome in Preterm Infants - 2013 Update. NEONATOLOGY 2013; 103(4):353-368.

16. Shane AL, Sánchez PJ, Stoll BJ: Neonatal sepsis. The Lancet 2017, 390(10104):1770-1780.

17. Berman Loren, Moss R Lawrence: Necrotizing enterocolitis: an update. Semin Fetal Neonatal Med 2011; 16: 145-150.

18. Duke T: Neonatal pneumonia in developing countries. Archives of Disease in Childhood - Fetal and Neonatal Edition 2005; 90(3):F211-F219.

19. Fink, Stacy: Intraventricular Hemorrhage in the Term Infant. Neonatal Network the Journal of Neonatal Nursing 2000; 19(7):13-18.

20. Lobmaier S M , A Müller, Huhn E, et al: HYPOXIC-ISCHEMIC ENCEPHALOPATHY. American Journal of Perinatology 2000; 17(03):113-120.

21. Salleh FHM, Zainudin S, Arif SM: Multiple Linear Regression for Reconstruction of Gene Regulatory Networks in Solving Cascade Error Problems. Advances in Bioinformatics 2017, 2017:1-14.

22. Implementation of the use of antenatal corticosteroids in the late preterm birth period in women at risk for preterm delivery. AM J OBSTET GYNECOL 2016, 215(2):B13-B15. 
23. Committee Opinion No. 713 Summary: Antenatal Corticosteroid Therapy for Fetal Maturation. Obstet Gynecol 2017; 130: 493-494.

24. Skoll A, Boutin A, Bujold E, Burrows J, Crane J, Geary M, Jain V, Lacaze-Masmonteil T, Liauw J, Mundle W et al: No. 364-Antenatal Corticosteroid Therapy for Improving Neonatal Outcomes. Journal of Obstetrics and Gynaecology Canada 2018; 40(9):1219-1239.

\section{Tables}

Table 1. Patient characteristics and clinical variables with and without ACS in the cohort. 


\begin{tabular}{|c|c|c|c|c|}
\hline & Total & Without ACS & With ACS & $P$-value \\
\hline $\mathbf{N}$ & 1472 & 672 & 800 & \\
\hline \multicolumn{5}{|l|}{ Mother } \\
\hline Age (years) & $28 \rrbracket 25-32 \rrbracket$ & $28 \rrbracket 25-31 \rrbracket$ & $29 \llbracket 25-32 \rrbracket$ & 0.008 \\
\hline Delivery & & & & 0.026 \\
\hline Vaginal & 846 & 409 (60.86\%) & 437 (54.63\%) & \\
\hline Cesarean & 626 & 263 (39.14\%) & 363 (45.37\%) & \\
\hline GDM & 77 & $32(4.76 \%)$ & 45 (5.63\%) & 0.459 \\
\hline PROM & 572 & 199 (29.61\%) & $373(46.62)$ & $\bowtie 0.001$ \\
\hline Placenta & & & & 0.795 \\
\hline Normal & 1291 & 591 (87.95\%) & 700 (87.50\%) & \\
\hline Abnormal & 181 & 81 (12.05\%) & $100(12.50 \%)$ & \\
\hline MSAF & 85 & 42 (6.25\%) & 43 (5.38\%) & 0.485 \\
\hline MG & 172 & 75 (11.16\%) & 97 (12.13\%) & 0.566 \\
\hline \multicolumn{5}{|l|}{ Infants } \\
\hline Gender & & & & 0.356 \\
\hline Female & 662 & 311 (46.28\%) & 351 (43.88\%) & \\
\hline Male & 810 & 361 (53.72\%) & 449 (56.12\%) & \\
\hline \multirow[t]{2}{*}{ GA } & 35.714 & 36 & 35.428 & $\otimes 0.001$ \\
\hline & (34.857-36.286) & $(35.286-36.571)$ & $(34.714-36.142)$ & \\
\hline BW (kg) & $2.37 \rrbracket 2.1-2.6 \rrbracket$ & $2.4 \llbracket 2.16-2.65 \rrbracket$ & $2.35 \llbracket 2.1-2.55 \rrbracket$ & $\varangle 0.001$ \\
\hline Asphyxia & 123 & $64(9.52 \%)$ & $59(7.38 \%)$ & 0.138 \\
\hline PS & 88 & 33 (4.91\%) & 55 (6.88\%) & 0.113 \\
\hline HIE & 339 & $150(22.32 \%)$ & $189(23.63)$ & 0.554 \\
\hline $\mathrm{NICH}$ & 40 & 20 (2.98\%) & $20(2.50 \%)$ & 0.576 \\
\hline NRDS & 136 & 64 (9.52\%) & $72(9.00 \%)$ & 0.730 \\
\hline NEC & 22 & $9(1.34 \%)$ & $13(1.63 \%)$ & 0.653 \\
\hline NS & 276 & $117(17.41 \%)$ & 159 (19.88\%) & 0.228 \\
\hline NP & 107 & 44 (6.55\%) & $63(7.88 \%)$ & 0.329 \\
\hline
\end{tabular}




\begin{tabular}{|lllll|}
\hline $\mathrm{NH}$ & 307 & $124(18.45 \%)$ & $183(22.88 \%)$ & 0.037 \\
\hline RS & 107 & $51(7.59 \%)$ & $56(7.00 \%)$ & 0.664 \\
\hline HE & 12083.96 & $9(4-14)$ & $10(5-15)$ & 0.001 \\
& $(7049.11-17918.58)$ & $(5983.46-17032.46)$ & $(7684.90-18281.83)$ & \\
\hline \multirow{2}{*}{ DHC } & 1201.44 & 1213.22 & 1192.76 & 0.002 \\
& $(980.19-1424.52)$ & $(969.45-1429.41)$ & $(986.5-1412.38)$ & \\
\hline
\end{tabular}

Results are shown as the median (min-max) or $\mathrm{n}(\%) . \otimes P \otimes 0.05$, indicates significant differences between the two groups. BW: Birth weight; DHC: Daily hospitalization cost; GA: Gestational age; GDM: Gestational diabetes mellitus; HE: Hospitalization expenses; HIE: Hypoxic-ischemic encephalopathy; LHS: Length of hospital stay; MG: Multiple gestation; MSAF: Meconium-stained amniotic fluid; NEC: Necrotizing enterocolitis of newborn; $\mathrm{NH}$ : Neonatal hypoglycemia; $\mathrm{NICH}$ : Neonatal intracranial hemorrhage; NP: Neonatal pneumonia; NS: Neonatal sepsis; PS: Pulmonary surfactant; RS: Respiratory support.

Table 2. Logistic regression for the neonatal complications in late preterm infants $(95 \% \mathrm{Cl})$ 


\begin{tabular}{|c|c|c|c|c|c|c|c|}
\hline & NRDS & $\mathrm{NH}$ & NS & NP & HIE & NEC & $\mathrm{NICH}$ \\
\hline \multicolumn{8}{|l|}{ Mother } \\
\hline Age & $\begin{array}{l}0.950- \\
1.045\end{array}$ & $\begin{array}{l}1.002- \\
1.054\end{array}$ & $\begin{array}{l}0.960- \\
1.016\end{array}$ & $\begin{array}{l}1.009- \\
1.091\end{array}$ & $\begin{array}{l}0.978- \\
1.032\end{array}$ & $\begin{array}{l}0.938- \\
1.109\end{array}$ & $\begin{array}{l}0.967- \\
1.096\end{array}$ \\
\hline ACS & $\begin{array}{l}0.332- \\
0.906\end{array}$ & $\begin{array}{l}0.812- \\
1.393\end{array}$ & $\begin{array}{l}0.726- \\
1.327\end{array}$ & $\begin{array}{l}0.635- \\
1.514\end{array}$ & $\begin{array}{l}0.895- \\
1.581\end{array}$ & $\begin{array}{l}0.356- \\
2.241\end{array}$ & $\begin{array}{l}0.478- \\
1.800\end{array}$ \\
\hline Delivery & $\begin{array}{l}0.874- \\
2.307\end{array}$ & $\begin{array}{l}0.925- \\
1.570\end{array}$ & $\begin{array}{l}0.527- \\
0.957\end{array}$ & $\begin{array}{l}0.525- \\
1.229\end{array}$ & $\begin{array}{l}0.792- \\
1.381\end{array}$ & $\begin{array}{l}0.458- \\
2.703\end{array}$ & $\begin{array}{l}0.151- \\
0.679\end{array}$ \\
\hline PROM & - & - & $\begin{array}{l}2.090- \\
3.816\end{array}$ & $\begin{array}{l}0.878- \\
2.073\end{array}$ & $\begin{array}{l}0.559- \\
1.003\end{array}$ & $\begin{array}{l}0.291- \\
1.898\end{array}$ & - \\
\hline Placenta & - & - & - & - & - & - & $\begin{array}{l}0.261- \\
2.495\end{array}$ \\
\hline AFMS & - & - & - & $\begin{array}{l}0.245- \\
1.407\end{array}$ & $\begin{array}{l}1.120- \\
2.891\end{array}$ & - & $\begin{array}{l}0.559- \\
6.317\end{array}$ \\
\hline MS & - & - & - & $\begin{array}{l}0.704- \\
4.300\end{array}$ & $\begin{array}{l}0.667- \\
2.141\end{array}$ & - & $\begin{array}{l}0.234- \\
4.628\end{array}$ \\
\hline MG & - & $\begin{array}{l}0.862- \\
1.898\end{array}$ & - & & & - & - \\
\hline GDM & - & $\begin{array}{l}0.353- \\
1.337\end{array}$ & - & $\begin{array}{l}0.949- \\
4.138\end{array}$ & $\begin{array}{l}0.635- \\
2.166\end{array}$ & - & $\begin{array}{l}0.043- \\
2.519\end{array}$ \\
\hline \multicolumn{8}{|l|}{ Infant } \\
\hline Gender & - & - & - & $\begin{array}{l}0.749- \\
1.705\end{array}$ & $\begin{array}{l}0.937- \\
1.615\end{array}$ & - & $\begin{array}{l}0.769- \\
3.032\end{array}$ \\
\hline GA & $\begin{array}{l}0.387- \\
0.724\end{array}$ & $\begin{array}{l}0.580- \\
0.812\end{array}$ & $\begin{array}{l}0.844- \\
1.223\end{array}$ & $\begin{array}{l}0.544- \\
0.926\end{array}$ & $\begin{array}{l}0.832- \\
1.185\end{array}$ & $\begin{array}{l}0.211- \\
0.688\end{array}$ & $\begin{array}{l}0.584- \\
1.585\end{array}$ \\
\hline BW & $\begin{array}{l}0.539- \\
1.831\end{array}$ & $\begin{array}{l}0.552- \\
1.071\end{array}$ & $\begin{array}{l}0.380- \\
0.772\end{array}$ & $\begin{array}{l}0.641- \\
1.740\end{array}$ & $\begin{array}{l}0.584- \\
1.134\end{array}$ & $\begin{array}{l}0.540- \\
4.871\end{array}$ & $\begin{array}{l}1.155- \\
5.379\end{array}$ \\
\hline Asphyxia & $\begin{array}{l}0.863- \\
3.504\end{array}$ & $\begin{array}{l}0.672- \\
1.655\end{array}$ & $\begin{array}{l}1.735- \\
4.364\end{array}$ & $\begin{array}{l}1.164- \\
3.996\end{array}$ & $\begin{array}{l}6.259- \\
15.076\end{array}$ & $\begin{array}{l}0.187- \\
3.940\end{array}$ & $\begin{array}{l}1.162- \\
7.624\end{array}$ \\
\hline RS & - & - & $\begin{array}{l}0.587- \\
2.339\end{array}$ & $\begin{array}{l}0.360- \\
2.072\end{array}$ & - & - & - \\
\hline PS & - & - & $\begin{array}{l}0.642- \\
2.644\end{array}$ & - & - & - & - \\
\hline NRDS & - & - & $\begin{array}{l}1.780- \\
5.763\end{array}$ & $\begin{array}{l}1.005- \\
4.558\end{array}$ & $\begin{array}{l}1.749- \\
3.948\end{array}$ & - & $\begin{array}{l}1.441- \\
7.029\end{array}$ \\
\hline $\mathrm{NH}$ & $\begin{array}{l}0.552- \\
1.771\end{array}$ & - & $\begin{array}{l}0.768- \\
1.522\end{array}$ & - & $\begin{array}{l}0.978- \\
1.855\end{array}$ & $\begin{array}{l}0.326- \\
2.532\end{array}$ & $\begin{array}{l}0.695- \\
3.209\end{array}$ \\
\hline NS & - & - & - & - & - & $1.023-$ & - \\
\hline
\end{tabular}




$\begin{array}{llllllll}\mathrm{NP} & - & - & 1.124- & - & - & 0.056- & - \\ & & & 2.846 & & & 3.305 \\ \mathrm{NEC} & - & - & 1.177- & - & - & -\end{array}$

ACS: antenatal corticosteroid; BW: Birth weight; DHC: Daily hospitalization cost; GA: Gestational age; HE: Hospitalization expenses; HIE: Hypoxic-ischemic encephalopathy; LHS: Length of hospital stay; MG: Multiple gestation; MSAF: Meconium-stained amniotic fluid; NEC: Necrotizing enterocolitis of newborn; $\mathrm{NH}$ : Neonatal hypoglycemia; NICH: Neonatal intracranial hemorrhage; NP: Neonatal pneumonia; NS: Neonatal sepsis; PS: Pulmonary surfactant; RS: Respiratory support.

Table 3. Linear regression for the length of hospital stay in late preterm infants (days).

\begin{tabular}{|c|c|c|c|c|c|}
\hline & Unstandardized B & Standardized coefficients beta & $P$-value & VIF & $\mathrm{F}$ \\
\hline Age & 0.096 & 0.057 & 0.005 & 1.053 & 105.354 \\
\hline ACS & -0.703 & -0.041 & 0.047 & 1.091 & \\
\hline Delivery mode & 1.312 & 0.077 & $\otimes 0.001$ & 1.075 & \\
\hline GA & -2.229 & -0.218 & $\nabla 0.001$ & 1.069 & \\
\hline BW & -6.981 & -0.351 & $\otimes 0.001$ & 1.178 & \\
\hline Asphyxia & 3.299 & 0.106 & $\nabla 0.001$ & 1.069 & \\
\hline NRDS & 4.919 & 0.165 & $\otimes 0.001$ & 1.087 & \\
\hline $\mathrm{NH}$ & 2.063 & 0.097 & $\otimes 0.001$ & 1.031 & \\
\hline NS & 4.565 & 0.207 & $\otimes 0.001$ & 1.082 & \\
\hline NP & 3.110 & 0.094 & $\nabla 0.001$ & 1.027 & \\
\hline HIE & 9.840 & 0.139 & $\otimes 0.001$ & 1.013 & \\
\hline
\end{tabular}

* $F 0.05=2.668 ; R^{2}=0.446$.

BW: Birth weight; GA: Gestational age; HIE: Hypoxic-ischemic encephalopathy; NH: Neonatal hypoglycemia; NP: Neonatal pneumonia; NS: Neonatal sepsis; 
Table 4. Linear regression for hospitalization expenses in late preterm infants (RMB).

\begin{tabular}{|c|c|c|c|c|c|}
\hline & Unstandardized B & Standardized coefficients beta & $P$-value & VIF & $\mathrm{F}$ \\
\hline ACS & -933.173 & -0.036 & 0.001 & 1.08 & 790.98 \\
\hline GA & -496.615 & -0.032 & 0.007 & 1.351 & \\
\hline BW & 1877.452 & 0.063 & $\bowtie 0.001$ & 1.37 & \\
\hline PS & 10555.095 & 0.193 & $₫ 0.001$ & 2.236 & \\
\hline RS & 4379.518 & 0.088 & $\varangle 0.001$ & 2.407 & \\
\hline NRDS & 3047.02 & 0.067 & $\otimes 0.001$ & 2.114 & \\
\hline $\mathrm{NH}$ & 2.08 & 0.098 & $\bowtie 0.001$ & 1.031 & \\
\hline NP & 2106.388 & 0.042 & $₫ 0.001$ & 1.048 & \\
\hline NS & 2247.55 & 0.068 & $\varangle 0.001$ & 1.136 & \\
\hline HIE & 833.192 & 0.027 & 0.014 & 1.139 & \\
\hline
\end{tabular}

* $F 0.05=2.668 ; R^{2}=0.844$.

ACS: antenatal corticosteroid; BW: Birth weight; GA: Gestational age; HIE: Hypoxic-ischemic encephalopathy; NH: Neonatal hypoglycemia; NP: Neonatal pneumonia; NS: Neonatal sepsis; PS: Pulmonary surfactant; RS: Respiratory support. 